\title{
MEDIAL PROFILES FOR MODELING DEFORMATION AND STATISTICAL ANALYSIS OF SHAPE AND THEIR USE IN MEDICAL IMAGE SEGMENTATION
}

\author{
GHASSAN HAMARNEH* \\ School of Computing Science, Simon Fraser University, \\ Burnaby, British Columbia V5A 1S6, Canada \\ hamarneh@cs.sfu.ca \\ http://www.cs.sfu.ca \\ RAFEEF ABU-GHARBIEH \\ Department of Electrical and Computer Engineering, University of British Columbia \\ Vancouver, British Columbia V6T 1Z4, Canada \\ rafeef@ece.ubc.ca \\ http://www.ece.ubc.ca \\ TIM MCINERNEY \\ Department of Computer Science, Ryerson University \\ Toronto, Ontario, M5B 2K3, Canada \\ tmcinern@scs.ryerson.ca \\ http://www.scs.ryerson.ca \\ Received (14 April 2003) \\ Revised (5 March 2004) \\ Accepted (11 November 2004) \\ Communicated by (Silvia Biasotti)
}

\begin{abstract}
We present a novel medial-based, multi-scale approach to shape representation and controlled deformation. We use medial-based profiles for shape representation, which follow the geometry of the structure and describe general, intuitive, and independent shape measures (length, orientation, and thickness). Controlled shape deformations (stretch, bend, and bulge) are obtained either as a result of applying deformation operators at certain locations and scales on the medial profiles, or by varying the weights of the main variation modes obtained from a new hierarchical (multi-scale) and regional (multi-location) principal component analysis of the medial profiles. We demonstrate the ability to produce controlled shape deformations on a medial-based representation of the corpus callosum. We show how this control of shape deformations facilitates the design of a layered framework for image segmentation and present results of segmenting the corpus callosum from $2 \mathrm{D}$ mid-sagittal magnetic resonance images of the human
\end{abstract}

${ }^{*}$ Corresponding author. 
brain. Furthermore we show how the medial-based representation facilitates hierarchical, deformation-specific statistical shape analysis of segmented corpora callosa.

Keywords: shape modeling; shape deformation; medial-based shape representation; principal component analysis; statistical shape analysis; image segmentation.

2000 Mathematics Subject Classification: 57N25, 62H25, 62H35.

\section{Introduction}

Controlling object shape deformations in a way that is based on intrinsic object geometry is highly desirable in image interpretation tasks, especially in the segmentation of biological objects from medical images. This intuitive deformation ability reflects the control over the molding of clay in a sculptor's hands and naturally lends itself to guidance by high-level controller mechanisms. Furthermore, performance of the controllers can be greatly enhanced by keeping deformations consistent with prior knowledge about possible object shape variations. Most deformable shape models $^{24}$ are boundary-based and although capable of local shape deformation, they lack the ability to undergo intuitive global deformation. As a result, in image segmentation applications it is difficult to incorporate intelligent deformation control operating at the right level of abstraction into the typical deformable model framework of energy minimization. Consequently, these models remain sensitive to initial conditions and spurious image features in image interpretation tasks.

Various hierarchical versions of boundary-based deformable models have been proposed $^{26,27,23,20}$. These methods however do not provide a natural global description of an object since their multi-scale deformation control is based on arbitrary boundary points rather than object-relative geometry. Several existing global or "volume-based" shape representation or deformation mechanisms $2,33,9,38,35$ are limited either by the type of objects they can model, or the type and intuitiveness of the deformations they can carry out. They are also typically not defined in terms of the object but rather the object is unnaturally defined (or deformed) in terms of the representation or deformation mechanism. Although deformation schemes that are independent of the underlying object geometry are beneficial when developing general deformation tools, they could be detrimental to shape extraction and analysis tasks.

A new set of deformable shape models founded upon the use of the medial axis transform or skeletonization ${ }^{4,21,1,5,6,34,11}$ are emerging as a powerful alternative to the earlier boundary-based and volume-based techniques. Medial based shape models provide both a local and a global description of shape. The deformations are defined in terms of an object's medial axis to allow natural and intuitive deformations that can be limited to a particular scale and location along the axis ${ }^{13,28,29,30,31}$

Deformable models that make use of statistical descriptions of the knowledge of shape variations have also been developed and applied to image segmentation ${ }^{7,37,8}$. These statistically-derived shape models relied on principal component analysis (PCA) and are only capable of producing global shape variation modes. 
Statistical analysis of medial based shape representation has been the focus of more recent research and applied to image segmentation, shape analysis, and feature selection ${ }^{39,19,40,36,22}$. Notable among the most recent research on this topic, with ideas dating back to the early sixties ${ }^{14}$, is the use of a geodesic component analysis, PGA (as opposed to PCA) for dealing with medial shape parameters that form Lie groups ${ }^{12}$.

In this paper, we propose medial-based profiles for shape representation and define deformation operators in terms of these shape profiles. Consequently we are able to intelligently control different types and extents of model deformations during model-to-data fitting processes. This ability allows the extraction of stable image features before proceeding to object regions with less well-defined features. To this end, we construct a model of an anatomical structure using a set of profiles derived from the medial axis of the structure, where each profile describes general and intuitive shape measures (length, orientation, and thickness). Structure deformations (stretch, bend, and bulge) are then implemented as deformation operators acting on the shape profiles, where each operator can have a different shape and scale and can be applied at any point along a profile. In addition to the general deformation operators, we utilize our knowledge about the object itself from a training set to generate statistically-proven feasible deformations. We control these statistical deformations locally along the medial shape profiles to support our goal of intelligent deformation scheduling. We develop spatially-localized feasible deformations at desired scales by utilizing hierarchical (multi-scale) and regional (multi-location) principal component analysis to capture shape variation statistics.

The remainder of the paper is organized as follows. In section 2 we begin by introducing medial-based shape profiles and describing their generation. Next, we describe the reconstruction of an object given it's medial profiles followed by a description of how a set of general operators act on the medial shape profiles to produce controlled shape deformations. We then describe how to perform statisticsbased deformations through the use of a multi-scale multi-location statistical analysis of the shape profiles (hierarchical regional principal component analysis, or HRPCA). Finally, we describe how operator- and statistics-based deformations are combined. In section 3 we describe the extraction of the medial profiles from the training set, present details on our algorithm for medial axis pruning, and demonstrate results of operator-, statistics-based, and combined deformations. We then present a hand-crafted segmentation schedule demonstrating the ability to control shape deformation. Next, we demonstrate the design of an automatic segmentation framework utilizing the underlying deformation capabilities. Finally, we present results of the multi-scale, deformation-specific statistical analysis of the segmented shapes. 


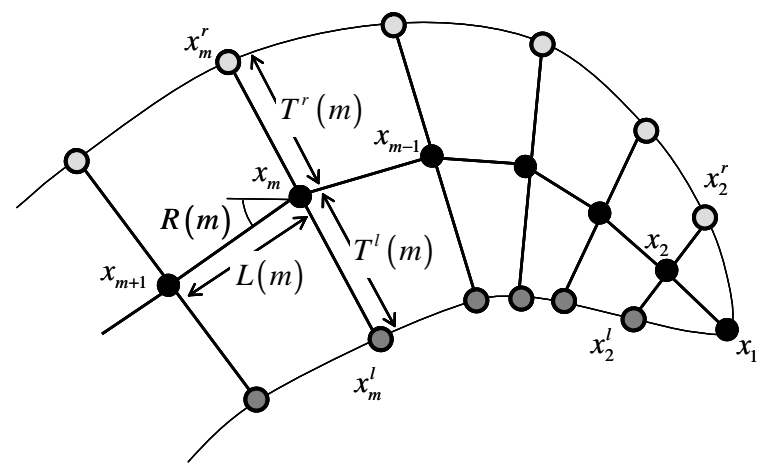

Fig. 1. Medial profiles shape representation. Medial nodes shown in black, left and right boundary nodes shown in dark and light gray, respectively. $x_{m}, x_{m}^{l}$ and $x_{m}^{r}$ are the $m^{t h}$ medial, left boundary and right boundary nodes, respectively. $L(m), R(m), T^{l}(m)$ and $T^{r}(m)$ are the length, orientation, left and right thickness profile values, respectively.

\section{Shape Representation and Deformation with Medial Profiles}

Controlling shape deformation requires a shape representation that, among other things, describes intuitive global shape characteristics. To meet this requirement, we represent shape with a set of rotation- and translation-invariant profiles that are based on a sampled medial axis of an object. Each profile captures an intuitive measure of shape: length, orientation, and thickness. Once the profiles are constructed, various deformation functions and/or operators can be applied producing intuitive, controlled deformations: stretching, bending, and bulging.

\subsection{Medial Profiles for Shape Representation}

We use a boundary representation of an object to generate the medial-based profiles. Generation of the profiles begins with the extraction of a sampled pruned skeleton of the object to obtain a set of medial nodes. Four medial profiles are constructed: a length profile $L(m)$, an orientation profile $R(m)$, a left (with respect to the medial axis) thickness profile $T^{l}(m)$, and a right thickness profile $T^{r}(m)$, where $m=1,2, \ldots, N, N$ is the number of medial nodes, and nodes 1 and $N$ are the terminal nodes. The length profile represents the distances between consecutive pairs of medial nodes, and the orientation profile represents the angles between segments connecting consecutive pairs of medial nodes. The thickness profiles represent the distances between medial nodes and their corresponding boundary points on both sides of the medial axis (Figure 1). Corresponding boundary points are calculated by computing the intersection of a line passing through each medial node in a direction normal to the medial axis, with the boundary representation of the object. Example medial profiles are shown in Figure 2. 

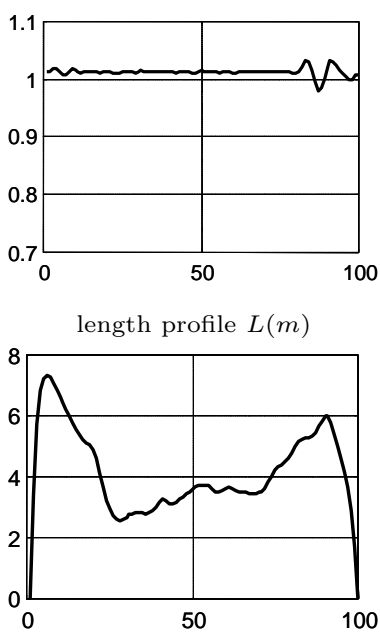

left thickness profile $T^{l}(m)$

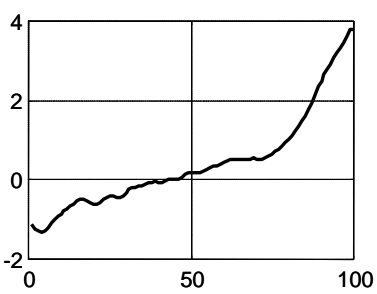

orientation profile $R(m)$

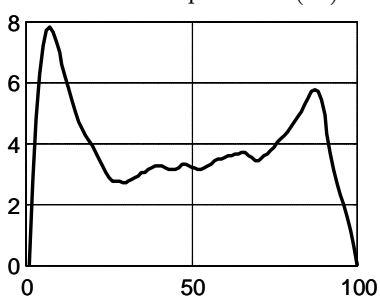

right thickness profile $T^{r}(m)$

Fig. 2. Medial shape profiles used to reconstruct the corpus callosum shown in Figure 3.

\subsection{Shape Reconstruction from Medial Profiles}

To reconstruct the object's shape given its set of medial profiles, we calculate the positions of the medial and boundary nodes by following the steps below.

(1) Specify affine transformation parameters: orientation angle $\theta_{\circ}$, translation values $\left(t_{x}, t_{y}\right)$, and scale values $\left(s_{x}, s_{y}\right)$.

(2) Using medial node 1 as the base or reference node, place it at location $x_{1}=$ $\left(t_{x}, t_{y}\right)$.

(3) Repeat steps 4 and 5 for $m=1,2, \ldots, N$.

(4) Compute the locations $x_{m}^{l}$ and $x_{m}^{r}$ of the left and right boundary points at either side of the $m^{t h}$ medial node (Figure 1) according to

$$
x_{m}^{l}=x_{m}+T^{l}(m)\left(\begin{array}{c}
s_{x} \cos \left(\theta_{\circ}+R(m)+\frac{\pi}{2}\right) \\
s_{y} \sin \left(\theta_{\circ}+R(m)+\frac{\pi}{2}\right)
\end{array}\right)
$$

and similarly,

$$
x_{m}^{r}=x_{m}+T^{r}(m)\left(\begin{array}{c}
s_{x} \cos \left(\theta_{\circ}+R(m)-\frac{\pi}{2}\right) \\
s_{y} \sin \left(\theta_{\circ}+R(m)-\frac{\pi}{2}\right)
\end{array}\right)
$$

(5) If $m<N$, compute the location $x_{m+1}$ of the next medial node $m+1$ as

$$
x_{m+1}=x_{m}+L(m)\left(\begin{array}{c}
s_{x} \cos \left(\theta_{\circ}+R(m)\right) \\
s_{y} \sin \left(\theta_{\circ}+R(m)\right)
\end{array}\right)
$$

Using the medial profiles of Figure 2 as an input to the previous algorithm we reconstruct the corpus callosum (CC) structure in Figure 3. Note that we have 


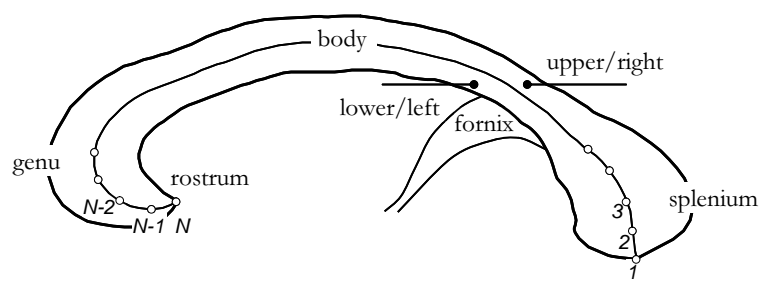

Fig. 3. Corpus callosum shape reconstruction resulting from the medial profiles in Figure 2.

generalized the reconstruction algorithm so that any medial node may serve as the base or reference node.

\subsection{Shape Deformations using Medial-Based Operators}

Once the shape profiles have been generated, we can construct deformation operators and apply them to the shape profiles. This results in intuitive deformations of the object upon reconstruction. That is, by applying an operator to the length, orientation, or thickness shape profile, we obtain a stretch, bend, or bulge deformation, respectively. Each deformation operator is implemented by defining a medial-based operator profile, $k(m)$, of a particular type ${ }^{3}$ (Figure 4 ) and specifying an amplitude, location, and scale values. The operator profile is then added to (or blended with) the medial shape profile corresponding to the desired deformation. For example, to introduce a bulge on the right boundary, an operator profile with a specific amplitude, type, location, and scale is generated and added to the right thickness medial profile $T^{r}(m)$ to obtain $T^{r}(m)+k(m), m=1,2, \ldots, N$ (Figure 5). In general, the application of a deformation operator $k_{d l s t}(m)$ alters the desired shape profile according to

$$
p_{d}(m)=\bar{p}_{d}(m)+\alpha_{d l s t} k_{d l s t}(m)
$$

where

$p$ is the shape profile

$d$ is the deformation type (stretch, bend, left/right bulge),

i.e. $p_{d}(m):\left\{L(m), R(m), T^{l}(m), T^{r}(m)\right\}$

$\bar{p}$ is the average (or original) shape profile

$k$ is the operator profile (with unity amplitude)

$l$ is the location (along the medial)

$s$ is the scale (or extent along the medial)

$t$ is the operator type (Gaussian, triangular, ..., etc.)

$\alpha$ is the operator amplitude.

Altering one shape profile only affects the shape property associated with that profile and does not affect any other object shape properties. For example, applying an operator to the orientation profile results in a bend deformation only and does 


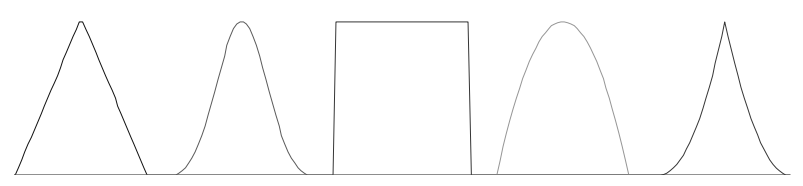

Fig. 4. Different types of deformation operators. From left to right: Triangular, Gaussian, flat, bell and cusp operators.

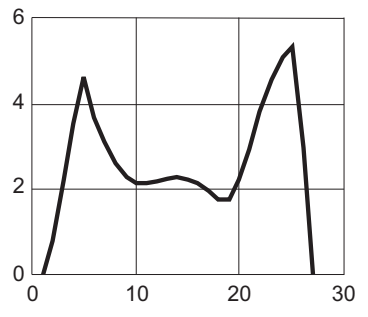

(a)

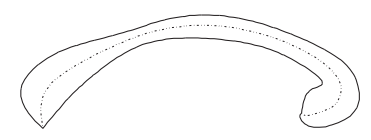

(c)

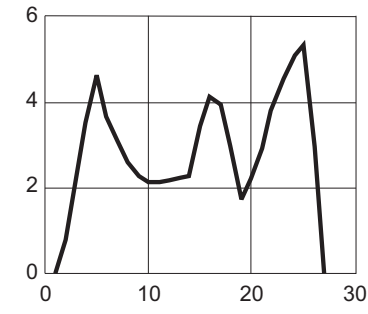

(b)

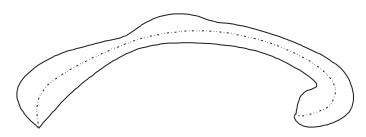

(d)

Fig. 5. Introducing a bulge on the $\mathrm{CC}$ upper boundary by applying a deformation operator on the upper thickness profile $T^{r}(m)$. (a) $T^{r}(m)$ before and (b) after applying the operator. (c) Reconstructed shape before and $(\mathrm{d})$ after applying the operator.

not result in a stretch or bulge. This implies the ability to perform successive operator-based object deformations of varying amplitudes, types, locations or scales, which can be expressed as

$$
p_{d}(m)=\bar{p}_{d}(m)+\sum_{l} \sum_{s} \sum_{t} \alpha_{d l s t} k_{d l s t}(m)
$$

In sections 3.4 and 3.5 we demonstrate the use of successive operator-based deformations in manual and automatic model-to-data fitting problems.

\subsection{Statistics-based Deformations using Hierarchical Regional Principal Component Analysis}

In many applications, prior knowledge about object shape variability is available or can be obtained by studying a training set of shape examples. The training set is typically created by labelling corresponding landmark points in each shape example. Principal component analysis (PCA) is then applied to the training set resulting in a point distribution model $(\mathrm{PDM})^{7}$. The PDM describes the average positions of 
the landmarks, the main modes of variation of landmark positions, and the amount of variation each mode explains. A drawback of this approach is that the result of varying the weight of a single variation mode generally causes all the landmark positions to change. In other words, although the original PDM model produces feasible shape deformations only, a desirable trait, it generally produces global deformations over the entire object. Our proposed method utilizes prior knowledge to produce feasible deformations while also controlling the scale and location of these deformations. Towards this end we perform a multi-scale (hierarchical) multilocation (regional) PCA on a training set of medial shape profiles. To achieve this, we collect spatially corresponding sub-profiles from the shape profiles. The length and position (extent) of a sub-profile reflect the scale and the location, respectively, over which the analysis is performed. The principal component analysis is now a function of the location, scale, and type of shape profile (length, orientation, or thickness). Thus, for each location, scale, and shape profile type, we obtain an average medial sub-profile, main modes of variation, and the amount of variation each mode explains. The result is that we can now generate a feasible (statisticsbased) stretch, bend, or bulge deformation at a specific location and scale.

Performing global (traditional) PCA on the medial profiles in a training set gives (for clarity, we have dropped the medial node index $m$ in the remainder of this section and adopted vector notation)

$$
\begin{gathered}
L=\bar{L}+M_{L} w_{L} \\
R=\bar{R}+M_{R} w_{R} \\
T^{l}=\bar{T}^{l}+M_{T^{l}} w_{T^{l}} \\
T^{r}=\bar{T}^{r}+M_{T^{r}} w_{T^{r}}
\end{gathered}
$$

where $L, R, T^{l}$ and $T^{r}$ are the medial profiles. $\bar{L}, \bar{R}, \bar{T}^{l}$ and $\bar{T}^{r}$ are the average profiles. The columns of the four $M$ matrices $\left(M_{L}, M_{R}, M_{T^{l}}, M_{T^{r}}\right)$ are the main modes of medial profile variation (for the length, orientation, left and right thickness profiles, respectively). Associated with each mode is the variance it explains. $w_{L}, w_{R}, w_{T^{l}}$ and $w_{T^{r}}$ are vectors of scalars weighing the different modes of variation. For a medial profile of length $N$ each of the four $M$ matrices is of size $N \times N$ and the average profiles and the weighing vectors are of length $N$. If the first $t$ modes of variation explain a sufficiently large portion of the variance (e.g. 99\%) in the training set, then the remaining modes can be ignored and the four $M$ matrices become of size $N \times t$ and the weighing vectors become of length $t$.

For capturing the shape variations at different locations and scales, we study the variations of the medial profiles in the desired localized region. Accordingly, the PCA becomes a function of the deformation type (length, orientation, left or right thickness), location, and scale (Figure 6). For example, to analyze the local variation in object length (stretch), we perform a statistical analysis on the length medial 


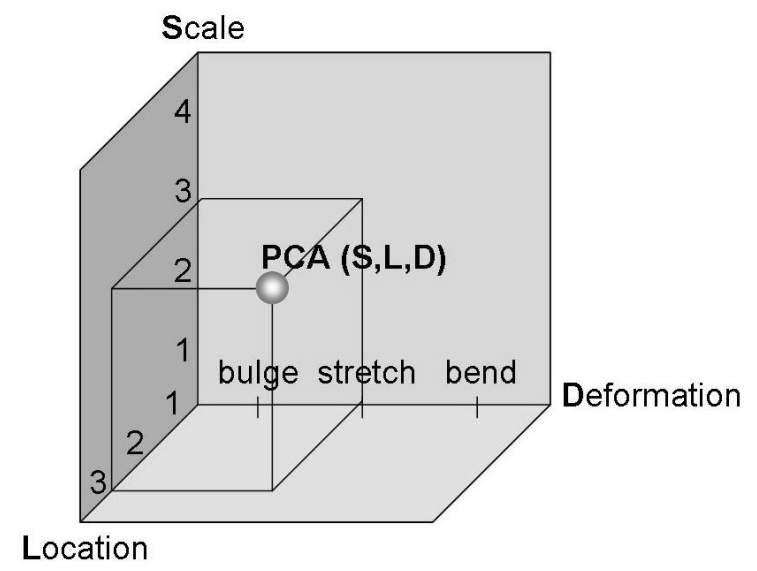

Fig. 6. Hierarchical regional principal component analysis. Here PCA is a function of the scale $(\mathrm{S})$, location $(\mathrm{L})$, and deformation $(\mathrm{D})$.

profile values of that local region. In general, for a single deformation-, location-, and scale-specific PCA we obtain the following model of medial profile variations,

$$
p_{d l s}=\bar{p}_{d l s}+M_{d l s} w_{d l s}
$$

where $p$ is the shape profile, $d$ is the deformation profile type, $l$ and $s$ are the location and scale values, $\bar{p}_{d l s}$ is the average medial profile, $M_{d l s}$ describes the main variation modes, and $w_{d l s}$ are weights of the variation modes and are typically limited to \pm 3 standard deviations. A more detailed description follows.

The deformation $d$ is either $L$ for length, $R$ for orientation, or $T^{l}\left(T^{r}\right)$ for left (right) thickness, i.e. $p_{d l s}:\left\{L_{l s}, R_{l s}, T_{l s}^{l}, T_{l s}^{r}\right\}$. The location and scale, $l$ and $s$, are measured w.r.t. the medial nodes and determine which values of the profile are to be included in the analysis according to

$$
p_{d l s}=\left[p_{d}(l), p_{d}(l+1), \ldots, p_{d}(l+s-1)\right] .
$$

For example, to analyze the variations of a right thickness bulge at location 'six' with scale 'four' (def,loc, scl $\left.=T^{r}, 6,4\right)$ in a training set we use

$$
p_{d l s}=p_{T^{r}, 6,4}=\left[T^{r}(6), T^{r}(7), T^{r}(8), T^{r}(9)\right],
$$

and for a bending deformation at location 'five' with scale 'three' (def, loc, scl = $R, 5,3)$ we have

$$
p_{d l s}=p_{R, 5,3}=[R(5), R(6), R(7)] .
$$

The average profile $\bar{p}_{d l s}$ is calculated according to

$$
\bar{p}_{d l s}=\frac{1}{n} \sum_{j=1}^{n} p(j)_{d l s}
$$


where $p(j)_{d l s}$ is $p_{d l s}$ obtained from the $j^{\text {th }}$ training example and $n$ is the number of training examples. The columns of $M_{d l s}$ are the eigenvectors, $e_{d l s}$, of the covariance matrix, $C_{d l s}$, and calculated by solving

$$
C_{d l s} e_{d l s}=\lambda e_{d l s}
$$

using

$$
C_{d l s}=\frac{1}{n-1} \sum_{j=1}^{n}\left(p(j)_{d l s}-\bar{p}_{d l s}\right)\left(p(j)_{d l s}-\bar{p}_{d l s}\right)^{T} .
$$

Note that the global PCA can be written as a special case of the HRPCA by specifying $l o c=1$ and $s c l=N$. Note also that for any shape profile type multiple variation modes can be activated, by setting the corresponding weighing factors to non-zero values, where each variation mode acts at a certain location and scale, hence we obtain

$$
p_{d}=\bar{p}_{d}+\sum_{l} \sum_{s} M_{d l s} w_{d l s}
$$

In summary, varying the weights of one or more of the variation modes alters the length, orientation, or thickness profiles and generates, upon reconstruction, statistically feasible stretch, bend, or bulge deformations at specific locations and scales.

\subsection{Combining Operator-and Statistics-Based Deformations}

In general, it is possible to combine operator- and statistics-based deformations (refer to (5) and (17)) using

$$
p_{d}=\bar{p}_{d}+\sum_{l} \sum_{s}\left(M_{d l s} w_{d l s}+\sum_{t} \alpha_{d l s t} k_{d l s t}\right) .
$$

A potential application of such combined deformations is the detection of pathology in anatomical structures. Towards that goal, statistics-based deformations can initially be used to fit the model to the overall expected shape of the structure, while operator based deformations are subsequently applied to match the model more accurately to the deviations from the normal. Note that several deformations, whether operator- or statistics-based, may spatially overlap (something that we currently do not restrict). Furthermore, adding profiles of different scales, hence different vector lengths, is possible by padding the profiles with zeros. Examples of combining operator- and statistics-based deformations are given in sections 3.3 and 3.4 .

\section{Implementation and Results}

We present implementation details and comprehensive results on the capabilities and performance of our method for shape representation, controlled deformation, 


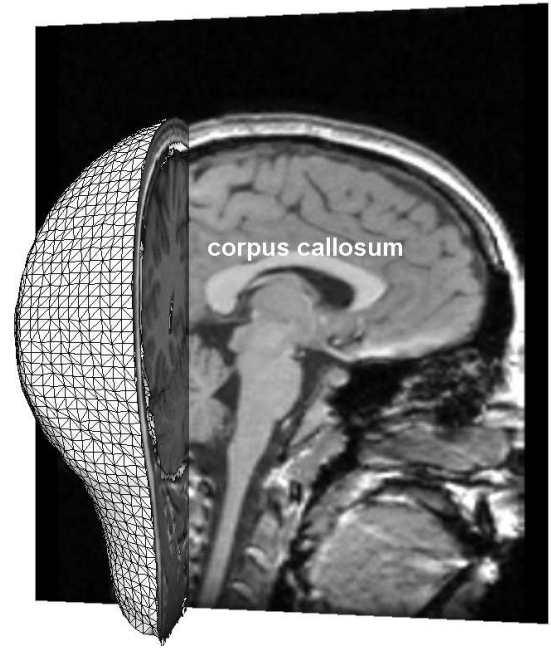

Fig. 7. Mid-sagittal slice of one of the 3D MR brain images. The corpus callosum is the bright ribbon-shaped object in the middle of the image.

segmentation, and (multi-scale and deformation-specific) statistical analysis of the corpus callosum $(\mathrm{CC})$ derived from $2 \mathrm{D}$ mid-sagittal magnetic resonance imaging (MRI) slices of the human brain. We first describe how the medial profiles were extracted from a training set followed by a description of the skeleton pruning algorithm used for medial axis extraction. We present results illustrating operatorbased, statistics-based, and combined deformations as well as a hand-crafted application illustrating intuitive and accurate control of shape deformation. We briefly discuss how we used higher level cognitive layers to control the deformation of an anatomical shape model represented by our medial shape profiles in order to achieve automatic medical image segmentation. We also demonstrate how the multi-scale, deformation-specific statistical analysis of the segmented structures is facilitated by the medial representation.

\subsection{Medial Profile Generation from Real Data}

From a set of 51 MRI brain volumes, we extracted the mid-sagittal slices from the coronal slices (Figure 7 and Figure 8(a)). We then used expert-segmented corpus callosum images (Figure 8(b)) to extract a set of boundary pixels ${ }^{15}$ (Figure 8(c)) and compute the set of spatially ordered boundary coordinates (Figure 8(d)). After extracting the skeleton using a standard technique ${ }^{16}$ (morphological operations to remove pixels on objects' boundaries without allowing them to break apart) (Figure $8(\mathrm{e})$ ), we calculated a pruned version (obtained as described in Section 3.2) to produce the primary medial axis (Figure $8(\mathrm{f})$ ). The primary medial axis is then sampled to obtain the coordinates of the medial nodes. 


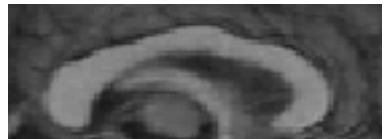

(a)

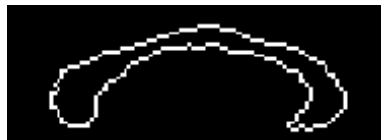

(c)

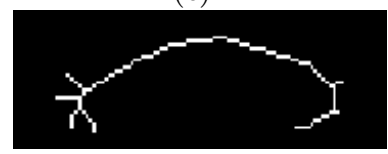

(e)

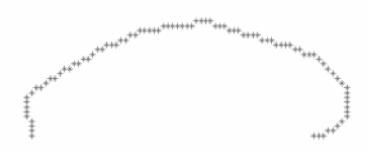

(g)

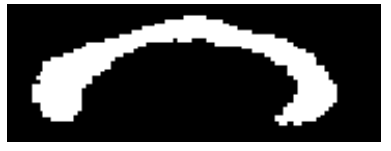

(b)

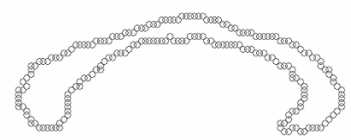

(d)

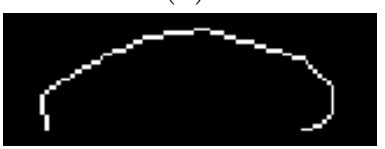

(f)

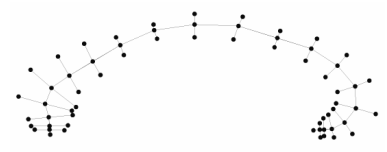

(h)

Fig. 8. Extracting the medial profiles mesh. (a) Close up of the CC, (b) manual CC segmentation, (c) boundary pixels and (d) coordinates, (e) skeleton (f) pruned skeleton (primary medial axis), (g) primary medial axis coordinates, and (h) medial and boundary nodes.

There are numerous alternatives to performing the medial sampling operation. Uniform or equal arc-length sampling can be carried out. Although this method is simple and easy to implement, it does not take into account the particular anatomy of the CC. Non-uniform sampling can be used to assign more medial points in different regions along the primary medial axis. Critical point detection algorithms ${ }^{41}$ or polygonal approximations ${ }^{18}$ can be utilized to sample medial nodes at geometrically significant locations. Hill and Taylor ${ }^{17}$ attempted to find corresponding points in a training set by minimizing the total variance of the resulting shape model. More recently there have been numerous research efforts to address the problem of automatically sampling corresponding points in a training set of shapes for building statistical models. Davies et al. ${ }^{10}$ optimized an information theoretic objective function (minimum description length criteria) to establish correspondence. Rueckert et $a l .{ }^{32}$ performed nonrigid registration by manipulating B-spline control points to obtain point correspondence prior to statistical analysis. Although a very important issue, the choice of method for automatic sampling is beyond the scope of this paper. The above mentioned alternatives will all yield a set of spatially ordered coordinates for the medial nodes. In this work we utilized nonuniform sampling with denser sampling allocated near the genu and splenium (Figure 8(g)). We then found the boundary points closest to the line normal to each sampled medial node (Figure $8(\mathrm{~h})$ ). From the locations of the medial and corresponding boundary nodes, 


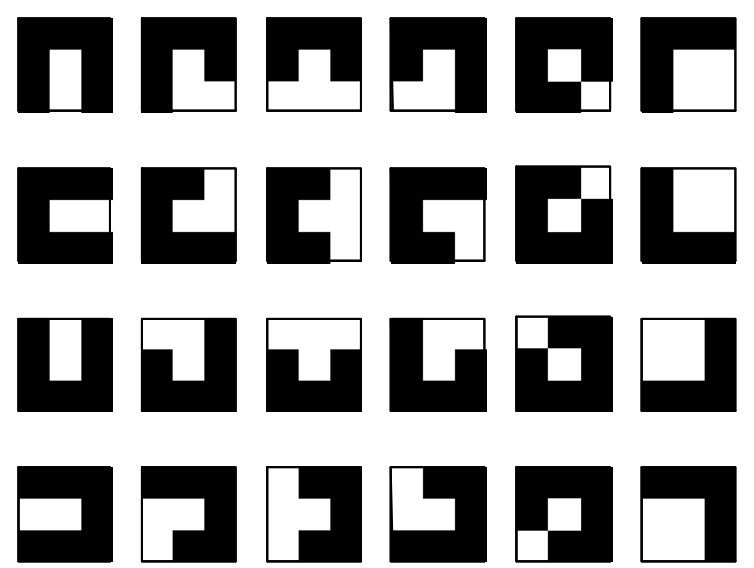

Fig. 9. The 24 terminal detection strels. Each column shows the four rotated versions of a single strel from Table 1.

we generated the medial shape profiles (as described in section 2.1) and performed medial-based HRPCA on the manual segmentation results (section 2.4).

\subsection{Skeleton Pruning for Medial Axis Extraction}

After extracting the skeleton of the binary expert-segmented images, we find all the skeleton branches by searching for terminal points or terminal pixels. Terminal pixels are located by utilizing hit-or-miss morphological operations ${ }^{15}$. We employ six $3 \times 3$ terminal detection structuring elements (strels) (Table 1) at each image pixel of the binary image containing the skeleton. For example, in the strel in Table 1(a), the mid-point corresponds to a terminal point pointing upwards. Three other strels are generated from each of the six strels by applying 90-degree rotations totaling 24 strels (Figure 9). Rotating the strel in Table 1(a) by 90 degrees counterclockwise gives a similar terminal-detection strel used for detecting terminal points that are directed to the left (leftmost column of Figure 9).

Table 1. The six terminal detection strels used in the skeleton pruning algorithm.

$\left[\begin{array}{lcc}0 & 0 & 0 \\ 0 & (1) & 0 \\ 0 & 1 & 0\end{array}\right] \quad\left[\begin{array}{lcc}0 & 0 & 0 \\ 0 & (1) & 0 \\ 0 & 1 & 1\end{array}\right] \quad\left[\begin{array}{ccc}0 & 0 & 0 \\ 0 & (1) & 0 \\ 1 & 1 & 1\end{array}\right] \quad\left[\begin{array}{ccc}0 & 0 & 0 \\ 0 & (1) & 0 \\ 1 & 1 & 0\end{array}\right] \quad\left[\begin{array}{ccc}0 & 0 & 0 \\ 0 & (1) & 0 \\ 0 & 0 & 1\end{array}\right] \quad\left[\begin{array}{ccc}0 & 0 & 0 \\ 0 & (1) & 1 \\ 0 & 1 & 1\end{array}\right]$

(a)

(b)

(c)

(d)

(e)

(f)

Each terminal point detected on the skeleton is considered to be the tip of one branch. Each branch is pruned with all other original branches still existing until 
the branch is so short that no terminal point is found. Pruning one branch after removing a previous branch gives unacceptable results since the location where the pruning of a single branch stops depends on where the other branches are. All locations of the pruned pixels are collected in a data structure and then removed all at once from the original skeleton image when all branches are pruned.

The extracted skeleton of the CC data we worked with consisted of a primary medial axis and branches on both the splenium and rostrum sides of the CC (Figure 3 ). These branches were pruned but the primary medial axis was not. Since these branches existed in all of the examples in our data set, we did not encounter a situation where the primary medial has an exposed terminal point and hence it was never pruned. In other structures (unlike our CC data) we may need to specify a maximum pruning length (maximum number of pruned pixels) beyond which the branch becomes significant (or possibly the primary medial axis itself) and is excluded from pruning.

\subsection{Operator-Based, Statistics-Based, and Combined Deformations}

This section presents shape deformation results based on the theory discussed in sections 2.3, 2.4, and 2.5. Figure 10a-d show examples of operator-based deformations while examples of statistics-based deformations are shown in Figure 10e-h. Figure $10 \mathrm{i}$ shows an example of combining operator- and statistics-based deformations.

\subsection{Hand-Crafted Application}

To demonstrate the capability and usability of the statistics- and operator-based controlled deformations, we handcrafted a deformation schedule for fitting the CC shape model to a mid-sagittal MRI slice of one of the brain images. Figure 11 shows the initial medial shape profiles and Figure 12 shows the resulting profiles after applying the fitting schedule. The initial and final CC shapes are shown in Figure 13. The schedule steps are shown in Table 2 and the resulting deformed CC shapes for each step of the schedule are shown in Figure 14.

\subsection{Automatic Image Segmentation}

We argue that intuitive shape deformation control is essential for the intelligent segmentation and high-level analysis of anatomical structures in medical images. We demonstrate this here through the use our proposed medial profiles in an automatic medical image analysis system. In Ref. 25, we introduced a new approach to medical image analysis that combines deformable model methodologies with concepts from the field of artificial life. In particular, we proposed "deformable organisms", autonomous agents whose task is the automatic segmentation, labelling, and quantitative analysis of anatomical structures in medical images. Analogous to natural organisms capable of voluntary movement, our artificial organisms possess deformable bodies with distributed sensors, as well as (rudimentary) brains 
(a)
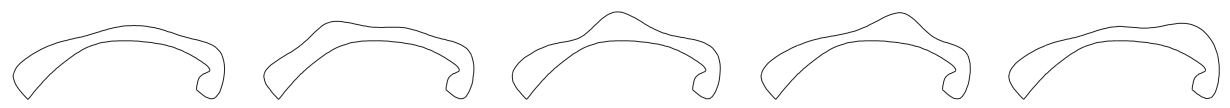

(b)
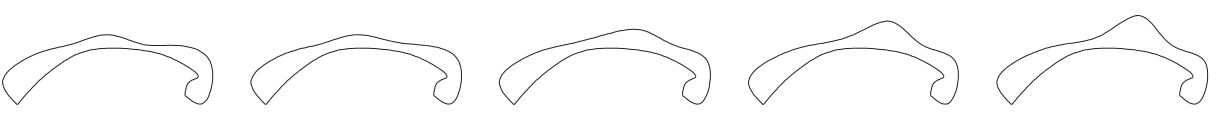

(c)
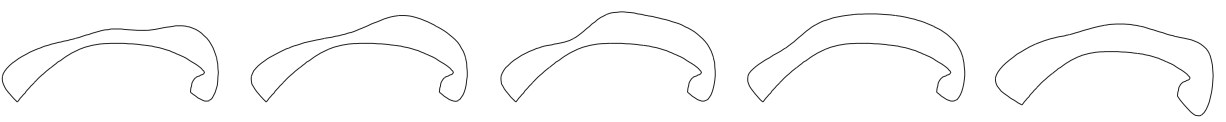

(d)
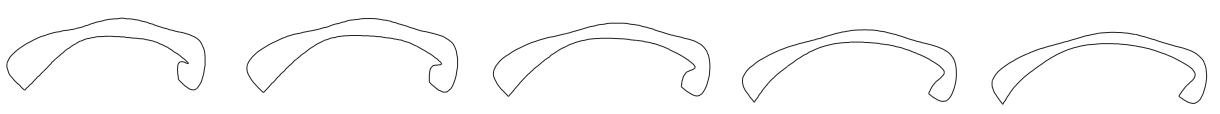

(e)
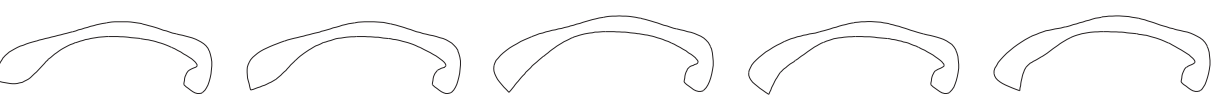

(f)
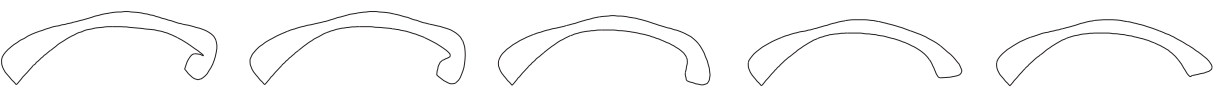

(g)
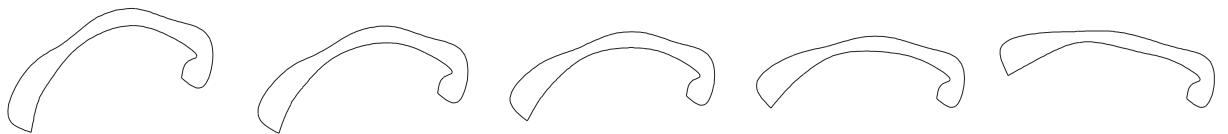

(h)
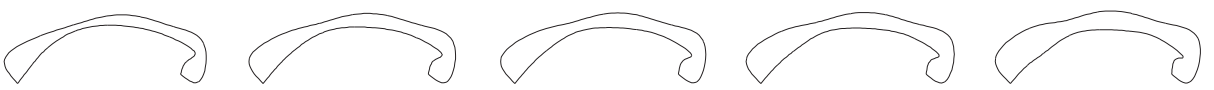

(i)
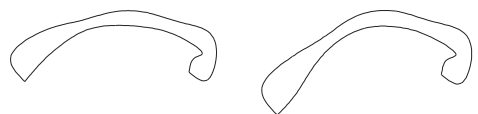

(1)

(2)

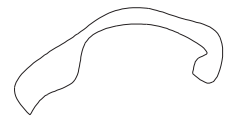

(3)

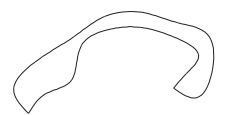

(4)

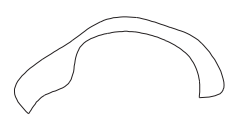

(5)

Fig. 10. Examples of controlled deformations: (a)-(c) Operator-based bulge deformation at varying locations, amplitudes and scales, respectively. (d) Operator-based stretching with varying amplitudes over entire CC. (e)-(g) Statistics-based bending of left end, right end, and left half of CC. (h) Statistics-based bulge of the left and right thickness over entire CC. (i) From left to right: (1) mean shape, (2) statistics-based bending of left half, followed by (3) locally increasing lower thickness using operator, followed by (4) applying operator-based stretch and (5) adding operator based bend to right side of CC.

with motor, perception, behavior, and cognition centers. Deformable organisms are perceptually aware of the image analysis process. Their behaviors, which manifest themselves in voluntary movement and alteration of body shape, are based upon sensed image features, pre-stored anatomical knowledge, and a deliberate cognitive plan. The medial-based shape profiles proposed in this paper were essential to the operation of the deformable organisms by providing potent and intuitive control of shape deformation by higher level controllers. 

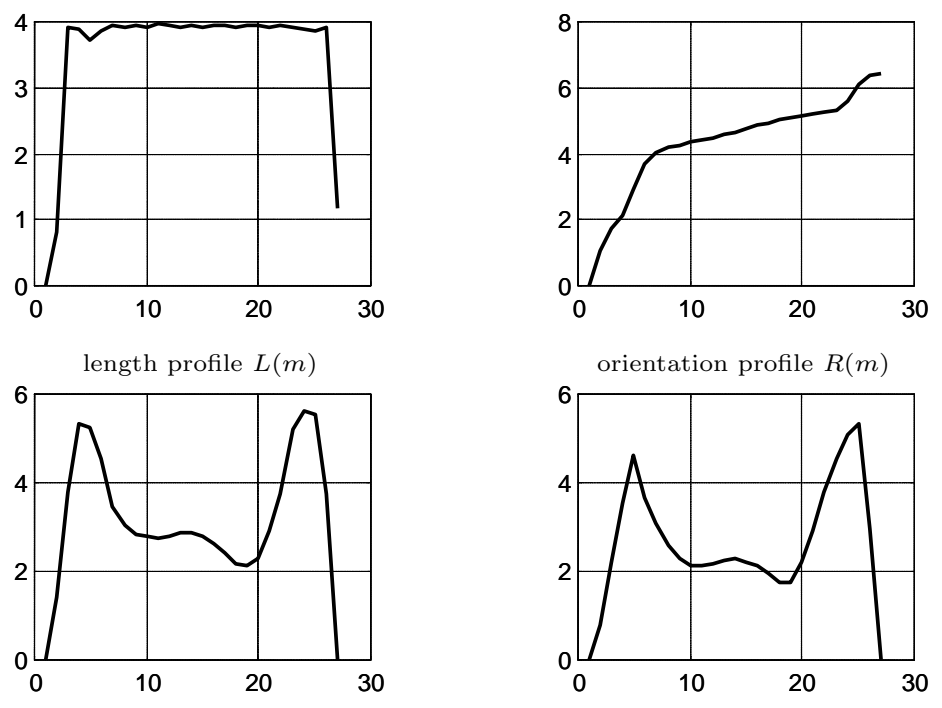

left thickness profile $T^{l}(m)$

right thickness profile $T^{r}(m)$

Fig. 11. Medial shape profiles of CC model before manual segmentation schedule.
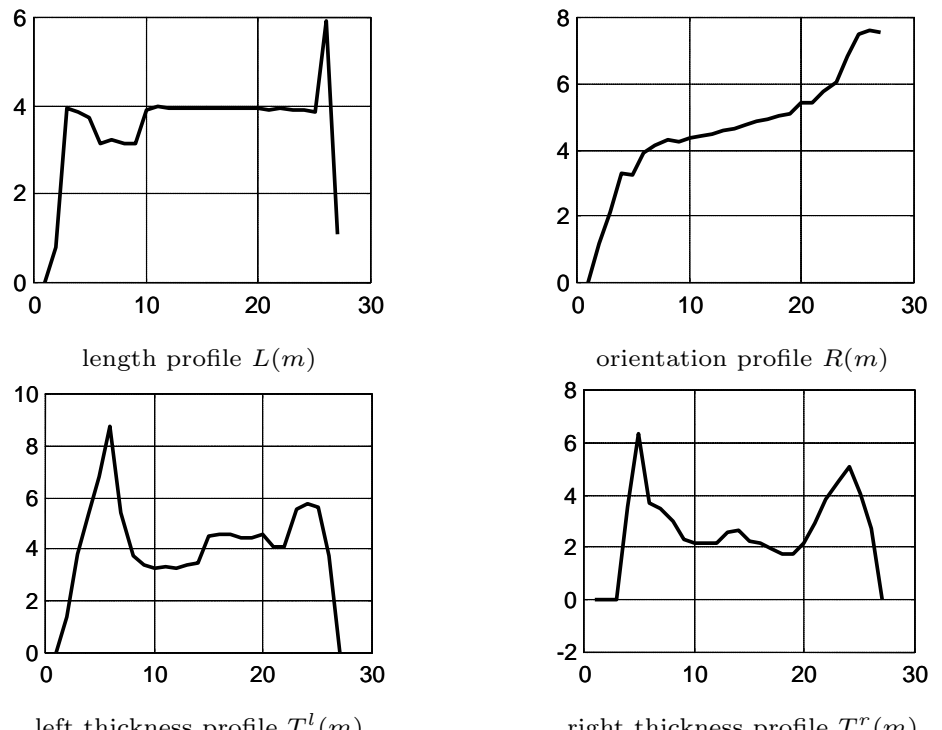

left thickness profile $T^{l}(m)$

right thickness profile $T^{r}(m)$

Fig. 12. Medial shape profiles of CC model after manual segmentation schedule.

In Ref. 25 we demonstrated several prototype deformable organisms based on a multi-scale axisymmetric body morphology, including a "corpus callosum worm" that can overcome noise, incomplete edges, considerable anatomical variation, and 


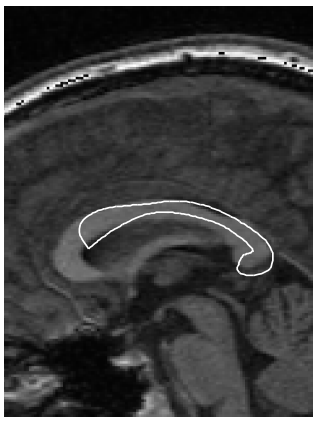

(a)

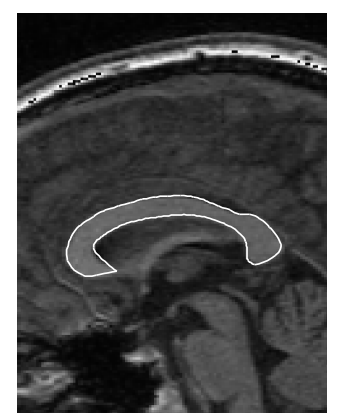

(b)

Fig. 13. Close up of the initial and final stages of the handcrafted fitting schedule.

Table 2. Deformation schedule used to fit the corpus callosum shape model to the MRI data.

\begin{tabular}{|c|c|c|c|c|c|}
\hline Step & Description & & & & \\
\hline 1 & Translation (7 & $\downarrow, 24 \rightarrow)$ & & & \\
\hline 2 & Rotation $10^{\circ}$ & $\mathrm{CW}$ & & & \\
\hline 3 & Scaling by 1.2 & & & & \\
\hline $\begin{array}{l}\text { Step } \\
\text { cont. }\end{array}$ & Deformation & Location & Scale & $\begin{array}{l}\text { Variation mode } \\
\text { or Operator type }\end{array}$ & $\begin{array}{l}\text { Variation mode weight } \\
\text { or Operator amplitude }\end{array}$ \\
\hline 4 & Bend & 1 & 8 & 2 & 0.50 \\
\hline 5 & Bend & 20 & 8 & 2 & -0.80 \\
\hline 6 & Bend & 22 & 6 & 2 & -0.75 \\
\hline 7 & Bend & 24 & 4 & 1 & 2.20 \\
\hline 8 & Bend & 1 & 4 & 2 & 1.00 \\
\hline 9 & Stretch & 6 & 4 & 1 & -1.50 \\
\hline 10 & Stretch & 26 & 1 & 1 & 2.00 \\
\hline 11 & Left-bulge & 15 & 7 & 1 & 3.00 \\
\hline 12 & Left-bulge & 18 & 3 & 1 & 2.00 \\
\hline 13 & Left-bulge & 6 & 12 & 1 & 3.00 \\
\hline 14 & Left-bulge & 5 & 3 & 1 & 3.00 \\
\hline 15 & Right-squash & 9 & 3 & 1 & -1.00 \\
\hline 16 & Right-bulge & 13 & 2 & 1 & 0.50 \\
\hline 17 & Left-bulge & 21 & 3 & Gaussian & 0.30 \\
\hline 18 & Left-bulge & 21 & 7 & Gaussian & 0.10 \\
\hline 19 & Right-squash & 24 & 2 & Gaussian & -0.50 \\
\hline 20 & Right-bulge & 4 & 2 & Bell & 1.70 \\
\hline 21 & Right-bulge & 6 & 3 & Gaussian & 0.40 \\
\hline 22 & Right-squash & 1 & 3 & Gaussian & -2.20 \\
\hline 23 & Right-squash & 25 & 1 & Gaussian & -0.80 \\
\hline
\end{tabular}



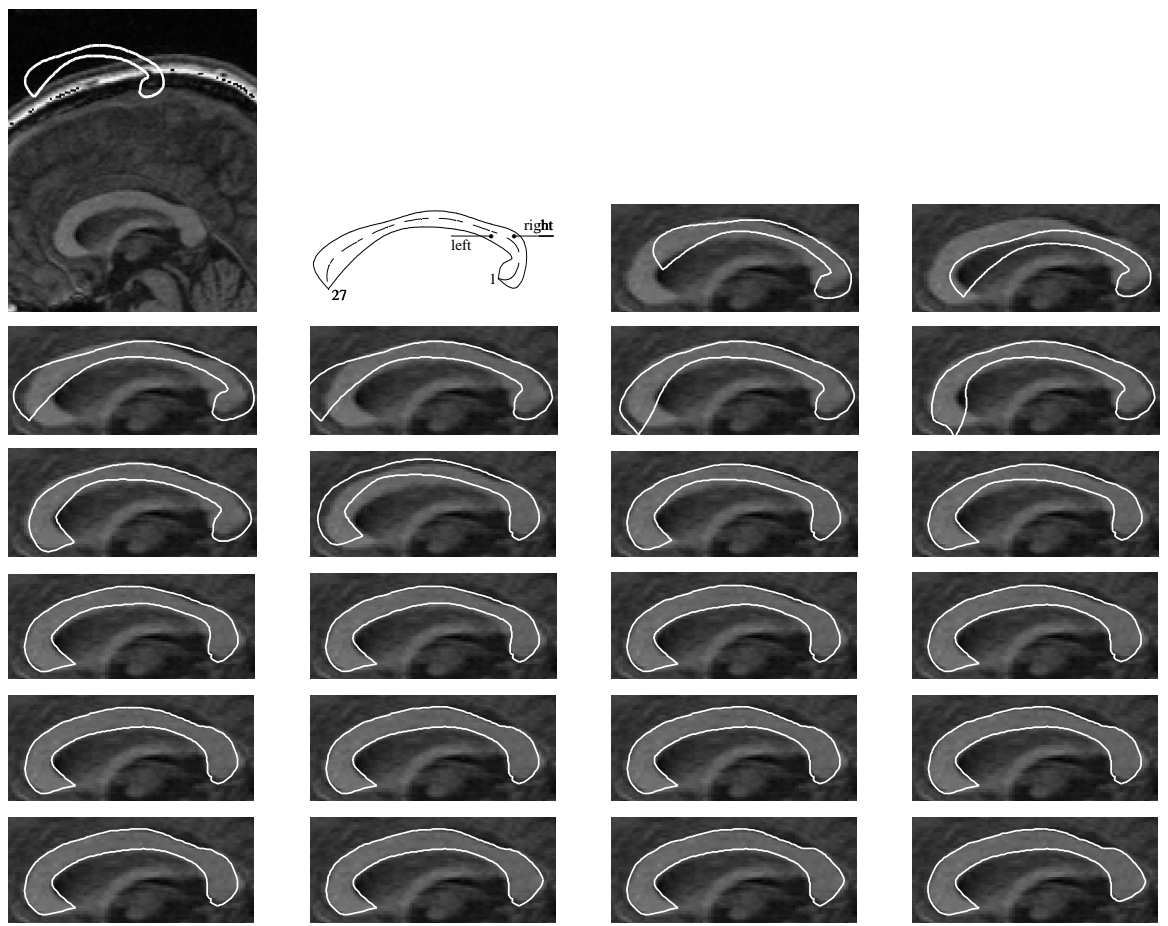

Fig. 14. Progress of the handcrafted fitting schedule (fitting steps are listed in Table 2).
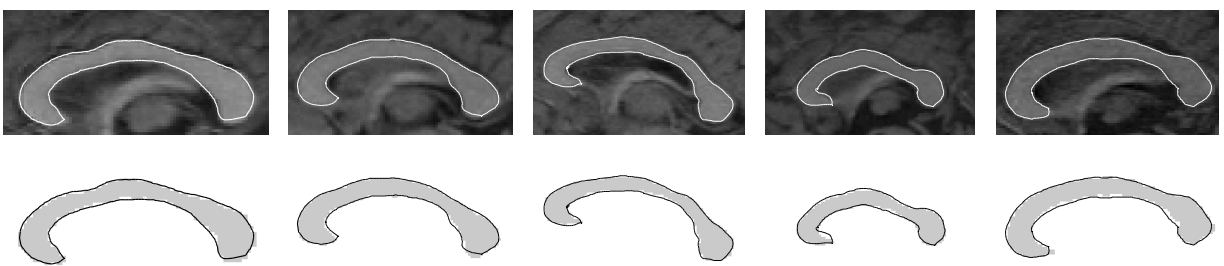

Fig. 15. Examples of automatic segmentation results (top), also shown (in black) over manually segmented (gray) CC (bottom).

interference from collateral structures to segment and label the corpus callosum in 2D mid-sagittal MR brain images (Figure 15). The principal technique adopted for segmenting the corpus callosum is the detection of CC features using a deformsense-decide routine. This is essentially done by deforming the shape model into a number of candidate configurations (by setting deformation parameters, such as the amplitude of a deformation operator (4) or a variation mode weight (10)), measuring image features and evaluating their agreement with predefined CC features, and finally deciding on the best configuration for fitting the model to the image data (Figure 16 and Figure 17). 

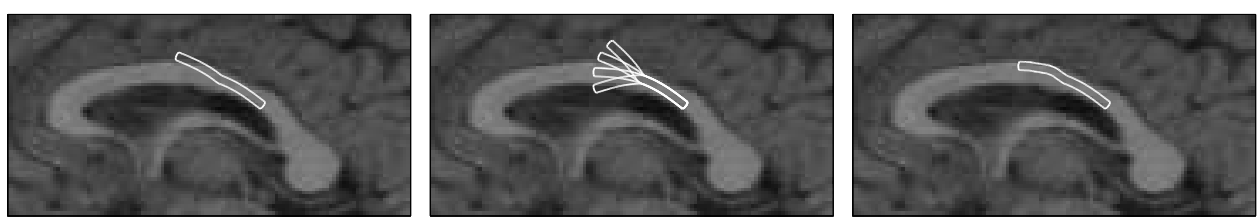

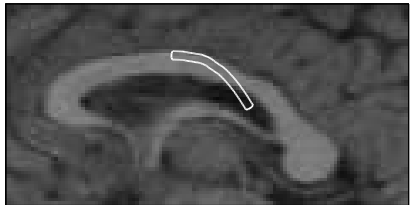

(a)

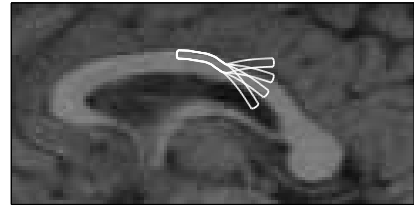

(b)

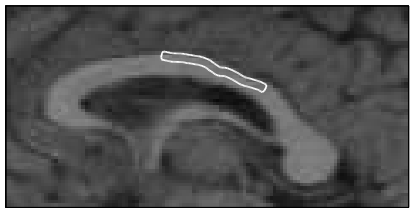

(c)

Fig. 16. The deform- sense-decide routine automatically tracking part of the upper CC boundary. (a) Initial shape, (b) deform and sense, (c) shape deformation decision.
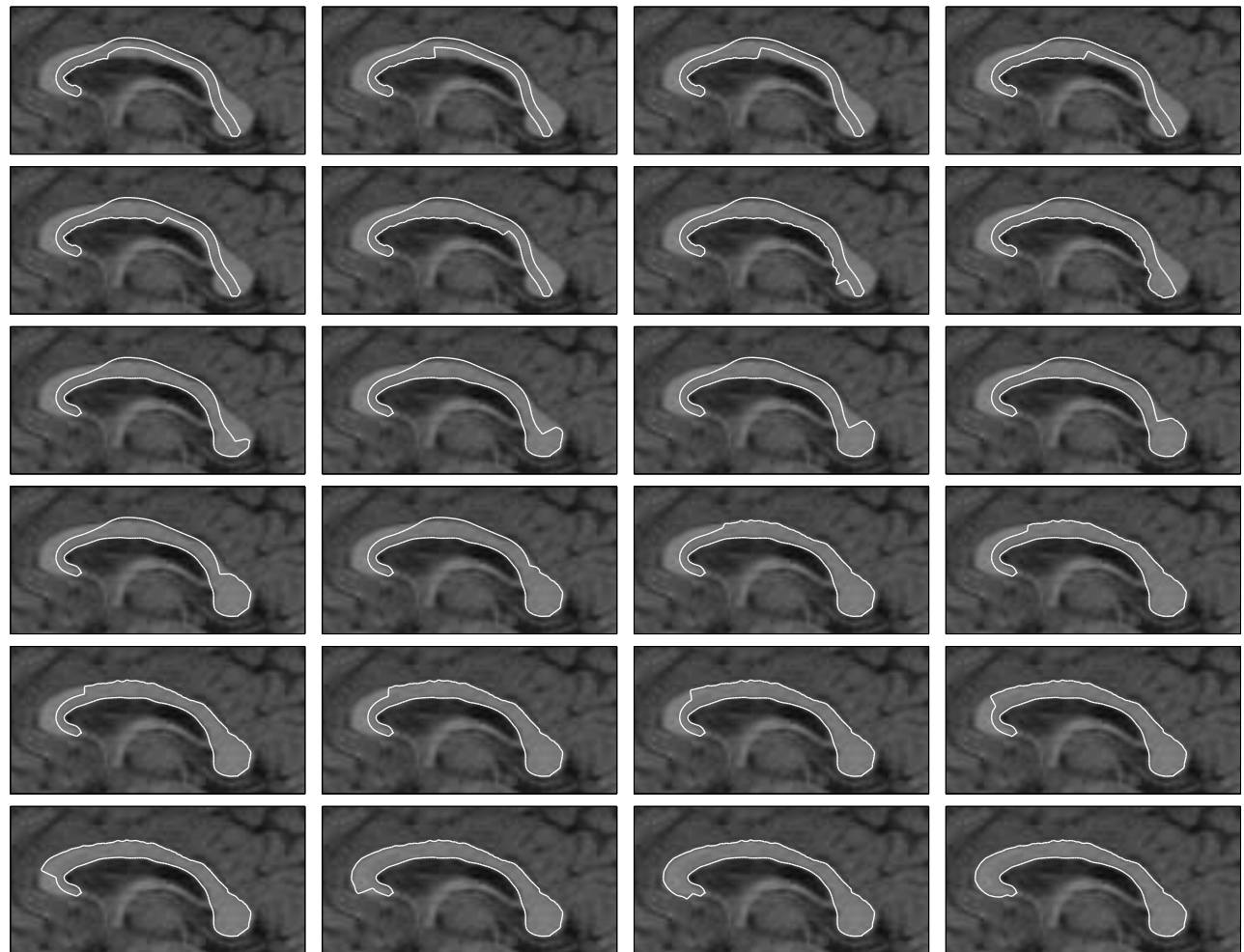

Fig. 17. Progress of automatically fitting the shape model to the lower CC boundary $\left(1^{\text {st }}\right.$ and $2^{\text {nd }}$ row) and to the upper boundary (remaining rows)

\subsection{Statistical Analysis of Automatically Segmented Structures}

We present results on the statistical analysis of 26 shapes automatically segmented as described in section 3.5. A "shape histogram", which is a gray-level image pro- 


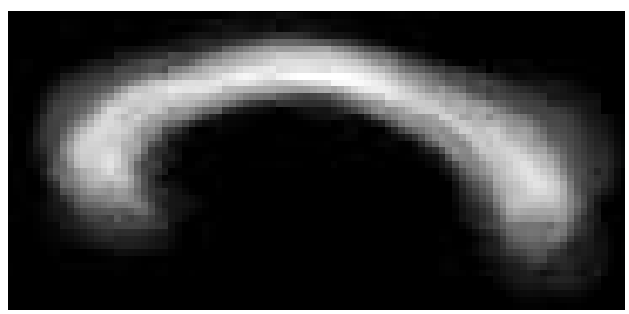

Fig. 18. Shape histogram (black=0, white $=25$ ) of all segmented CCs.

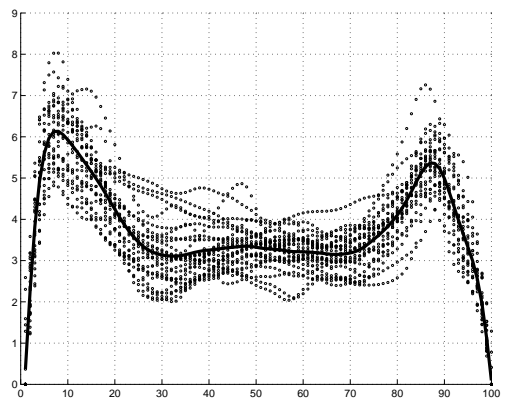

(a)

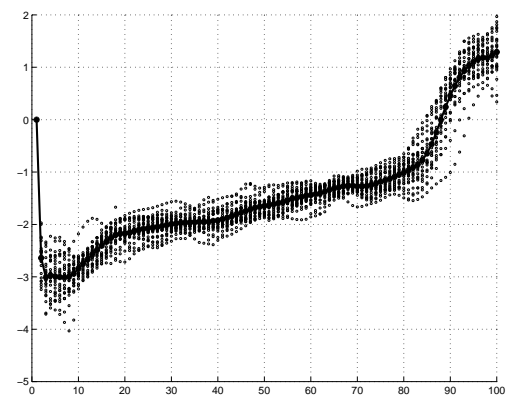

(b)

Fig. 19. Overlay plot of (a) thickness and (b) orientation profiles of all segmented CCs. Mean profiles appear in bold.

duced by superimposing the binary images of the segmented shapes after suitable alignment, is shown in Figure 18. The alignment we performed utilized translations in the $\mathrm{x}$ and $\mathrm{y}$ directions, resulting in equal first-order moments for all the shapes.

The shape histogram is one way to visualize the overall variability in the segmented CCs. It is apparent that shape variation exists with varying degrees and in different regions of the CC. Figure 19 further illustrates the variability using an overlay plot of the medial-based orientation and thickness profiles of the segmented CCs. The visualization of these profiles provide more insight into understanding the variations present in the data by identifying contributions of different types of deformations along the $\mathrm{CC}$.

In order to decompose these shape variations into intuitive deformations (stretching, bending, bulging) and to quantify their extent, location, and scale, we performed the proposed HRPCA on the medial-based shape profile representation of the segmented shapes, as described in section 2.4. To demonstrate these capabilities we examined the principal shape variation modes captured by HRPCA. We activated different modes of variations (type, location, scale) according to (17), then reconstructed and displayed the resulting shapes. Figure 20 shows examples of activating global and local main modes of stretching, bending, and boundary 


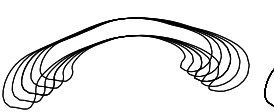

(a)

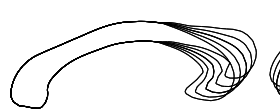

(b)

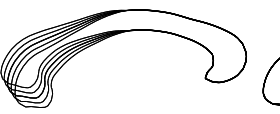

(c)

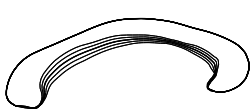

(d)

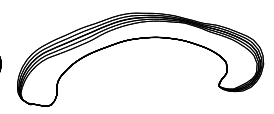

(e)

Fig. 20. Main mode of shape variation of the automatically segmented data: (a) global stretch, (b-c) local bend, global (d) lower and (d) upper boundary thickness.

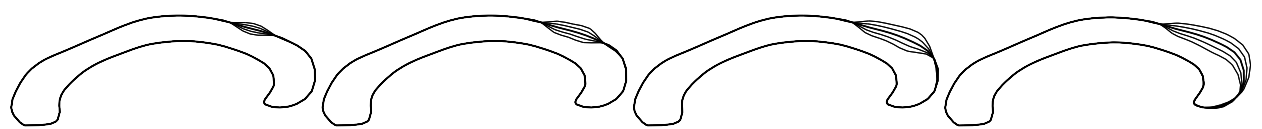

Fig. 21. Main modes of local upper thickness variations at varying scales.

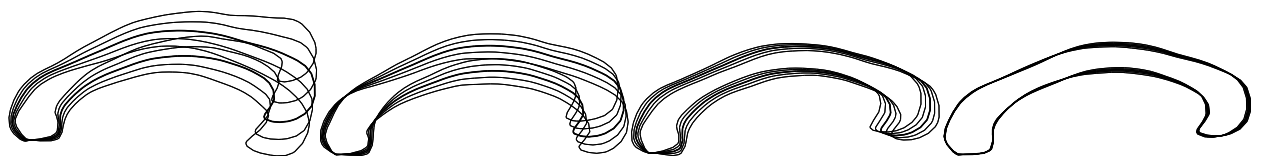

Fig. 22. From left to right: $1^{\text {st }}, 2^{\text {nd }}, 3^{\text {rd }}$, and $4^{\text {th }}$ main modes of global bending variation explaining $41.07,14.15,11.96,6.68 \%$ of the total variation, respectively.

thickness variations. Figure 21 shows the main modes of local upper thickness variations at different scales. The effect of varying the first four modes of global bending variations is shown in Figure 22.

\section{Conclusion}

We presented 'medial profiles', a medial-based shape representation that provides the ability to deform shapes in an intuitive (stretching, bending, bulging) and controlled (multi-scale, multi-location) manner. Furthermore, by introducing HRPCA, we were able to perform hierarchical and regional statistical analysis of the shape profiles and generate statistically feasible versions of the deformations. We included comprehensive results of the capabilities and performance of our method for shape representation, controlled deformation, automatic segmentation, and statistical analysis of the corpus callosum derived from 2D mid-sagittal magnetic resonance imaging slices of the human brain. We are currently working on extending the method to 3D and on linking multiple interacting medial-based shape models to represent more complex structures. Furthermore, we are investigating potential advantages of replacing the linear PCA with other statistical analysis tools that may capture more accurately the statistical variations of the medial profiles. 


\section{References}

1. D. Attali and A. Montanvert, Computing and Simplifying 2D and 3D Continuous Skeletons, Computer Vision and Image Understanding, 67(3) (1997) 261-273.

2. A. H. Barr, Global and Local Deformations of Solid Primitives, Proceedings of SIGGRAPH'84, 18(3) (1984) pp. 21-30.

3. J. R. Bill and S. K. Lodha, Sculpting Polygonal Models using Virtual Tools, Proceedings of Graphics Interface, (1995) pp. 272-279.

4. H. Blum, Biological shape and visual science, J. Theoretical Biology, 38 (1973) 205-287.

5. G. Borgefors, I. Nystrom and G. S. D. Baja, Computing skeletons in three dimensions, Pattern Recognition, 32, (1999) 1225-1236.

6. S. Bouix, P. Dimitrov, C. Phillips and K. Siddiqi. Physics-Based Skeletons, Proceedings of Vision Interface (2000) Montreal, Canada.

7. T. F. Cootes, D. Cooper, C. J. Taylor and J. Graham, Active Shape Models - Their Training and Application, Computer Vision and Image Understanding, 61(1) (1995) $38-59$.

8. T. F. Cootes, G. J. Edwards and C. J. Taylor, Active Appearance Models, IEEE PAMI, 23(6), (2001) 681-685.

9. S. Coquillart, Extended Free Form Deformations: A Sculpting Tool for 3D Geometric Modeling, Proceedings of SIGGRAPH'90, 24(2) (1990) 187-196.

10. R. H. Davies, C. J. Twining, T. F. Cootes, J. C. Waterton and C. J. Taylor, A Minimum Description Length Approach to Statistical Shape Modelling, IEEE TMI 21(5), (2002) 525-537.

11. P. Dimitrov, J.N. Damon and K. Siddiqi, Flux Invariants for Shape, Proceedings of Computer Vision and Pattern Recognition 1 (2003), 835-841.

12. P. T. Fletcher, L. Conglin, S. M. Pizer, S. Joshi, Principal geodesic analysis for the study of nonlinear statistics of shape, IEEE TMI, 23(8) (2004), 995- 1005.

13. D. Fritsch, S. M. Pizer, L. Yu, V. Johnson and E. Chaney, Segmentation of medical image objects using deformable shape loci, Proceedings of the International Conference on Information Processing in Medical Imaging, Lecture Notes in Computer Science, 1230 (Springer-Verlag, 1997) 127-140.

14. U. Grenander, Probabilities on Algebraic Structures, (John Wiley and Sons, 1963).

15. R. C. Gonzalez and R. E. Woods, Digital Image Processing (Prentice Hall, 2002).

16. R. M. Haralick and L. G. Shapiro, Computer and Robot Vision, Volume I, (AddisonWesley, 1992).

17. A. Hill and C. Taylor, Automatic landmark generation for point distribution models, Proceedings British Machine Vision Conference (1994), 429-438.

18. A. Jain, Fundamentals of Digital Image Processing (Prentice Hall, 1989).

19. S. Joshi, S. Pizer, P. T. Fletcher, P. Yushkevich, A. Thall and J. S. Marron, Multiscale Deformable Model Segmentation and Statistical Shape Analysis Using Medial Descriptions, IEEE TMI, 21(5) (2002) 538-550.

20. J. O. Lachaud and A. Montanvert, Deformable meshes with automated topology changes for coarse-to-fine three-dimensional surface extraction, J. Medical Image Analysis 3(1) (1999) 1-21.

21. F. Leymarie and M. D. Levine Simulating the grassfire transform using an active contour model, IEEE PAMI 14(1) (1992) 56-75.

22. C. Lu, S. M. Pizer and S. Joshi, A Markov Random Field Approach to Multi-scale Shape Analysis, International Conference on Scale Space Methods in Computer Vision, Lecture Notes in Computer Science, 2695 (Springer-Verlag, 2003) 416-431.

23. C. Mandal, B. C. Vemuri and H. Qin, A New Dynamic FEM-based Subdivision Surface Model for Shape Recovery and Tracking in Medical Images, Proceedings MICCAI'98, 
1496 (1998) 753-760.

24. T. McInerney and D. Terzopoulos, Deformable Models in Medical Image Analysis: A Survey, J. Medical Image Analysis 1(2) (1996) 91-108.

25. T. McInerney, G. Hamarneh, M. Shenton and D. Terzopoulos, Deformable Organisms for Automatic Medical Image Analysis, J. Medical Image Analysis, 6(3) (2002) 251266.

26. J. V. Miller, D.E. Breen, W.E. Lorensen, R. M. O'Bara and M.J. Wozny, Geometrically Deformed Models: A Method for Extracting Closed Geometric Models from Volume Data, Proceedings of SIGGRAPH'91, 25(4)(1991) 217-226.

27. J. Montagnat and H. Delingette, Volumetric medical image segmentation using shape constrained deformable models, Proceedings of Computer Vision, Virtual Reality and Robotics in Medicine, (1997) 13-22.

28. S. M. Pizer, D. S. Fritsch, K. C. Low and J. D. Furst, 2D \& 3D Figural Models of Anatomic Objects from Medical Images, Mathematical Morphology and Its Applications to Image Processing, eds. H.J.A.M. Heijmans, J.B.T.M. Roerdink (Kluwer Computational Imaging and Vision Series, Amsterdam, The Netherlands 1998) 139-150.

29. S. M. Pizer and D. S. Fritsch, Segmentation, Registration, and Measurement of Shape Variation via Image Object Shape, IEEE TMI, 18(10) (1999) 851-865.

30. S. M. Pizer, G. Gerig, S. Joshi and S. R. Aylward, Multiscale medial shape-based analysis of image objects, Proceedings of the IEEE, bf 91(10) (2003), 1670-1679.

31. S. M. Pizer, Medial \& Medical: A Good Match for Image Analysis, International Journal of Computer Vision, Special Issue on Research at the University of North Carolina, Medical Image Display \& Analysis Group, 55(2-3) (2003) 79-84.

32. D. Rueckert, A. F. Frangi and J. A. Schnabel, Automatic construction of 3D statistical deformation models of the brain using non-rigid registration, IEEE TMI, 22(8) (2003) 1014-1025.

33. T. W. Sederberg and S. R. Parry, Free-Form Deformation of Solid Geometric Models, Proceedings of SIGGRAPH'86, 4(20) (1986) 151-160.

34. K. Siddiqi, S. Bouix, A. TannenBaum and S. Zucker, Hamilton-Jacobi Skeletons International Journal of Computer Vision 48(3), (2002) 215-231.

35. K. Singh and E. Fiume, Wires: A Geometric Deformation Technique, Proceedings of SIGGRAPH'98, 99(1) (1998) 405-414.

36. M. Styner, G. Gerig, J. Lieberman, D. Jones and D. Weinberger, Statistical shape analysis of neuroanatomical structures based on medial models, J. Medical Image Analysis, $\mathbf{7 ( 3 )}$ (2003) 207-220.

37. G. Szekely, A. Kelemen, Ch. Brechbuehler and G. Gerig, Segmentation of 3D objects from MRI volume data using constrained elastic deformations of flexible Fourier surface models, J. Medical Image Analysis, 1(1) (1996) 19-34.

38. D. Terzopoulos and D. Metaxas, Dynamic 3D models with local and global deformations: deformable superquadrics, IEEE PAMI 13(7) (1991) 703-714.

39. P. Yushkevich, S.M. Pizer, S.Joshi and J.S. Marron, Intuitive, Localized Analysis of Shape Variability, Proceedings of the International Conference on Information Processing in Medical Imaging, Lecture Notes in Computer Science, 2082 (Springer-Verlag, 2001) 402-408.

40. P. Yushkevich, S. Joshi, S. M. Pizer, J. G. Csernansky, and L.E. Wang, Feature Selection for Shape-Based Classification of Biological Objects, Proceedings of the International Conference on Information Processing in Medical Imaging, Lecture Notes in Computer Science, 2732 (Springer-Verlag, 2003) 114-125.

41. P. Zhu and P. M. Chirlian, On Critical Point Detection of Digital Shapes, IEEE PAMI, 17(8) (1995) 737-748. 